\title{
Exploring Industry 4.0 Paradigm as Applied to Project Management: A Proof of Concept
}

\author{
Paolo Eugenio Demagistris, Waseem Khan \\ Politecnico di Torino, Turin, Italy
}

\begin{abstract}
This research study elaborates and conceptualizes the architectural framework of Industry 4.0 to be applied to project management. Research is based on the literature in digital transformation in project management and extends the literature in adding the feasibility of Industry 4.0 applied to project management. The exploring paradigm of Industry 4.0 is 5-layer architecture. Mixed research approach, a blend of qualitative and quantitative approach is adopted with the objective of acquisition of knowledge, examination of the concepts, and analysis of the theories in order to understand feasibility of Industry 4.0 in a constructive research methodology. Findings of the research suggest the feasibility of implementation of Industry 4.0 concepts for project management, especially for project monitoring and control. It is explored that there can be an automation of project management process with a certain degree of capability in decision making.
\end{abstract}

Keywords: project management, project management 4.0, CPS architecture, project automation, digital EVA

\section{Introduction}

Project performance management is very crucial for success of a project, performance management regulates expectations for employee performance and that keeps them motivated to work in such a way that is demanded by the project nature. For managers the method to increase the work performance and effectiveness, time monitoring and control is a very powerful tool for the overall project performance, which leads to data acquisition and its effective interpretation to land on a better decision.

In and on time data interpretation and decision making is very important for performance evaluation, for a project manager or project team, and for this purpose a certain level of automation is required. Manufacturing sectors have already coped with the issues that emerged automation in processes and called it Industry 4.0.

The Industry 4.0 is the fourth industrial revolution, which refers to the integration of several emerging technologies including Internet of Thing (IoT), Big Data/Analytics, Cyber Security, Cloud Computing, Cognitive Computing, Machine to Machine Communication systems, RFID Technology, and Advanced Robotics. This disruptive technology mainly focuses on the real time data, machine learning, interconnectivity of devices and automation of processes.

Paolo Eugenio Demagistris, B.Eng (Hons), M.Sc.Eng., C.Eng (Italy), LCI (UK), SFC, professor of Practice of Project Management, Department of Management and Production Engineering, Politecnico di Torino, Turin, Italy.

Waseem Khan, MS (Engineering Management), Department of Management and Production Engineering, Politecnico di Torino, Turin, Italy.

Correspondence concerning this article should be addressed to Paolo Eugenio Demagistris, or Waseem Khan, Department of Management and Production Engineering, Politecnico di Torino, Corso Duca Degli Abruzzi, 24, 10129, Turin, Italy. 
If we consider, generally fourth industrial revolution "Industry 4.0 " is a broader domain that takes production processes, efficiency, data management, relationship with customers, competitiveness, and much more within its boundary. Specifically, Industry 4.0 has become the new theme for management.

This advanced innovation is rising achievements in various fields, for example, mechanical autonomy, AI, Nano-innovation, quantum computing, biotechnology, the Internet of Things (IoT), 3D printing, Augmented Reality and self-governing vehicles, frameworks, fabricating.

Numerous modern chiefs anticipate that the Industry 4.0 will convey uncommon dimensions of development and profitability over the coming decades.

At its hearth Industry 4.0 is about deploying a stack of technology which enables cyber-physical systems deployment. This means that as Industry 4.0 allows the creation of new business models, the same PP\&PM may develop different methodological frameworks. The paradigm of cyber-physical systems is the orchestration of processes by integrating data repositories with IoT and IoP. The internet of people through hand held, wearable or recognition devices by which people can interact through apps with nodes of IoP itself or the IoT, to achieve a data driven execution system. Data collection and analysis, also trough machine learning, is allowed to track transformations, be them material, as in manufacturing and construction, or immaterial, as information or knowledge creation. Eventually this will lead to a full set of analytical capabilities: descriptive, diagnostic, predictive, prescriptive.

Industry 4.0 is generally the interest of research these days in educational institutions, and they are making future generation able to adopt the trending technology, as well as industries also focusing on the trend evolving rapidly, and wanting to increase productivity and ease in operations.

Project management these days is effective but not efficient, a blog written with authentic sources (Danielle, 2016), statistics on projects claim that $70 \%$ of projects fail due to ill performance and communication gaps, and that only $40 \%$ of projects at IBM meet the company's three key goals (schedule, budget, and quality), furthermore 57 percent of projects fail due to breakdown in communications.

It's now time to introduce project management with latest technological innovation, so that this sector also gets benefit from new trends and overcomes that problems and challenges which can be addressed with the implementation and application of Industry 4.0 in the line of action in project management transforming the process model and increase efficiency.

\section{Research GAP}

Scanning the research in the area of I4.0, it is evident that the integration of cyber physical system is increasing very rapidly in the production and manufacturing sectors. They have identified the goal of I4.0 as optimization of the value chains by adopting and implementing such phenomenal procedures and technology which can help us in autonomously controlling and dynamic production.

Strange and Zucchella (2017) highlight the development of I4.0 will greatly affect the activities and strategic decisions of multinational enterprises. The MNEs should control the structure and distribution phases of the global value chains. Further it is reported that 3-D printing innovation and virtual building will conceivably enable clients to give contribution on product plan and impact choices on where and when the product is to be manufactured. The improvement of BDA and IoT are distinguished as the capabilities that will control authoritative execution later on. 
According to Preuveneers and Ilie-Zudor (2017), the 4th industrial revolution based on Cyber-Physical Systems can monitor, analyze, and automate business processes, transforming production, and logistic processes into smart factory environments where big data capabilities, cloud services, and smart predictive decision support tools are used to increase productivity and efficiency.

Win and Kham (2018) drew basic concept of I4.0 and its implementation in project management, and they also highlighted challenges for project management in I4.0, project managers roles, role of cloud computing, IoT and other basic aspects, however their research could not explain architecture for implementation and were not able to define the working procedure of I4.0 for project management monitoring and control. Kolberg and Zuhlke (2015) presented basic architecture for lean manufacturing in I4.0 concept, and they established a concept how I4.0 should be integrated into already available lean systems. They also claimed the fact that lean system is natural precursor for I4.0. on the other hand, Lee, Bagheri, and Hung-An (2015) proposed a cyber-physical system architecture for I4.0 in the area of manufacturing systems, and they modeled a 5C architecture which is also known as 5-layer pyramidal architecture, with layers for smart connections, data to information conversions, cyber integration, cognition and configuration. This work could be adopted for project management with the help of IoT, cloud computing systems, and other data generation devices.

Oesterreich and Teuteberg (2016) stated that cloud computing, mobile computing, and modularization are matured in terms of market to utilize them as Industry 4.0 components while additive manufacturing, robotics and AR/VR are still under development.

Concepts of Industry 4.0 need to be elaborated in order to discuss the feasibility of these concepts in the field of project management and development of the architecture for ICT infrastructure for the implementation of I4.0 in project monitoring and control.

Theorin, Bengtsson, Provost, Lieder, Johnsson, Lundholm, and Lennartson (2017) in their conceptual paper, considering current factory infrastructure they studied the rapid integration of smart services, and presented LISA (line information system architecture), which is very innovative and simple. Although this research is dedicated to production factories, its concepts are of great worth for project monitoring.

Muller, Kiel, and Voigt (2018) investigated the relations between I4.0 opportunities and challenges in context of sustainability. They claimed in their work that strategic, operational, environmental, and social opportunities are favorable for sustainable implementation of I4.0.

The 5-layer architecture of Industry 4.0, according to Lee et al. (2015), also builds upon third layer which is cyber physical system, that consists of mathematical algorithms pre formulated equations setups to get the desired kind of results. Every industrial sector has some mathematics and KPIs to measure and evaluate the success of its business, industry 4.0 has became crucial for problem management, like Li, Y. Wang, and K. S. Wang (2017) worked upon formulating a systematic approach to get knowledge about Industry 4.0 based predictive maintenance addressing processes of fault analysis and treatment for predictive maintenance in machineing centers. Project management is determined to manage and measure project based on earned value method. The key for successful implementation of I4.0 in project management would be EVM mathematical algorithms based on cyber-physical system.

Derivations and descriptions of earned value analysis and management are although completely entertained in a lot of sources in different perspectives, including Project Management Institute (PMI), around a decade ago, if we take PMI as our base reference, the standard practice of PMI published in 2005, differentiate 
EVM in two classes, first the key parameters (planned value, earned value, and actual cost), and EVM measures including variances, indices, and forecasts (Anabri, 2003).

According to Hunter, Fitzgerald, and Barlow (2014), EVA is the value provider to the project management team; in the manner that it enhances the capability to monitor costs and schedules, and helps in foresee expected problems, corrective plans and implementation of corrective strategies. The key factor for EVMS success is the capability of working team in planning, monitoring, and controlling the project in correct balance between process and outcomes. EVA environment is effective for cost and schedule problem (Narbaev \& Demarco, 2013).

According to Mahadik and Bhangale (2013), EVA is improvement against traditional accounting system; it helps the management to see potential risks in time. It is considered as the early alarming tool for project managers, enabling them to recognize and control issues before they become a liability, it is a tool to manage better, on time and on allocated budget.

According to Preuveneers and Ilie-Zudor (2017), business sectors are shifting from traditional wired infrastructure to innovative wireless networks for the purpose to continuously monitor the performance of their plant operations, keeping in view previously techniques which were so much complicated to expand and very rigid in terms of rapidly evolving to production market dynamics. Wireless technologies offer clear advantages to monitor production assets and corresponding network manufacturing or logistics processes.

Petrasch and Hentschke (2016) claim that IoT devices are not used for their own sake, they must contribute to more effective or a more efficient business process or a better user experience, furthermore all major architectural and methodological frameworks include process modeling, TOGAF, and ADM (Architectural Development Method). A business process model makes it possible to consider I4.0 aspects like IoT devices on a non-technical level for business modeling introducing a modeling notation.

The question arises, while project management community is concerned about considering Industry 4.0 implementation in project management and automated system for decision making in projects, which is supposed to generate and interpret data on its own with the help of devices and produce reports and forecast final results, the literature is blank about the feasibility of the concepts in project management, and no business process model for I4.0 implementation for project monitoring and control and no information on the architecture of PM4.0 available in the research in the subject domain.

\section{Methodology}

This paper seeks to answer to two most important questions in this context:

- Is it practically feasible to implement paradigms of Industry 4.0 in project management?

- What will be the practical business process model and architecture of ICT infrastructure of project management Industry 4.0?

In order to address the research question, this research is basically a constructive approach research, which means in this work focus is kept on the application of Industry 4.0 concepts in possible areas of project management so that projects can be managed and monitored in a more advanced and efficient way to ensure the performance and productivity of project in the near future, this work also consists of explanation of employee time monitoring techniques with Industry 4.0 procedure and an explanatory analysis of time expenditure for allocated task to employee, with the help of famous project management procedure for evaluation of projects performance, which is Earned Value Analysis. 
Algorithms are formulated to access project performance in relation to the employee time allocation array, and Earned Value Analysis is the core of Industry 4.0 which is Cyber Physical System, these algorithms are to be used to design a CPS system for the I4.0 based monitoring and control system for project management.

This research is based on quasi experimental research methodology, which means this research looks like experimental research but is not actually experimental research based on real data. Although data used in this research are supposed data according to the real life scenarios and conditions, which regard the constructive approach of research. The independent variable which is employee time allocation for allocated task is manipulated, keeping other variables baseline constant.

The research approach of this work is a blend of qualitative and quantitative, because this paper is aimed at the acquisition of knowledge, examination of the concepts, and analysis of the theories in order to understand feasibility of Industry 4.0 in project management and interpretations of data to prove the feasibility of concept.

Following data used for the evaluation:

- Employee Time Expenditure on task per day;

- Baseline of project task per day;

- Employee fixed payment, either daily or monthly basis;

- Deadlines;

- Project or task planned budget.

This research is based on qualitative as well as quantitative research approach because this work is aimed at the acquisition of knowledge, examination of the concepts, and analysis of the theories in order to understand feasibility of Industry 4.0 in project management and interpretations of data to prove the feasibility of concept. The broad quantitative approach could also be adopted for the research, but to due unavailability of capable resources for the acquisition of real data in order to access real information, qualitative approach is widely adopted, on the other hand quantitative approach is being utilized in a narrow scope.

\section{Sources of Information}

Primary data. Primary data on time expenditure of employee on the allocated task have been supposed for the purpose of evaluation and exploration of the concept, time supposition is based on the real life scenarios, and common time utilization patterns followed by average employee on projects.

Apart from that Task Planned Value (BCWS), Task Duration had also been supposed for exploration and lies in the borders of primary data.

Table 1

Primary Data

\begin{tabular}{ll}
\hline Task allocated to employee & Process design \\
\hline Tools & Design software \\
\hline Deadline & 8 days max \\
\hline Total required effort & 64 hours \\
\hline Employee cost & $800 /$ day \\
\hline
\end{tabular}

Secondary data. Secondary data about average employee time utilization on allocated task are obtained from the online researches, according to research on daily employee time expenditure, around $40 \%$ of time is being theft everyday on average. 
According to US Pay Roll Association, time theft affects about 75\% of business, creating a loss of around 400 Billion \$ per year. It is considered a silent killer of efficiency.

Data collected from time tracking device or any other mean can be utilized for earned value analysis of project.

This research is based on the primary data only as the replacement of tracking device, bypassing the first step of 5-layer architecture of I4.0 which is data acquisition. The framework of 5-layer can be effectively examined with bypassing the first layer if time allocation (data) is known already. Here we supposed the data for exploration of paradigm and determine the feasibility.

On the other hand, we have some privacy regulation concerns and limitations as well in assessing personal data of employee or employee time allocation on tasks during projects with the help of data acquisition devices, imposed by the European General Data Protection Regulations (GDPR-2016). The GDPR significantly affects businesses in all industry sectors, including those outside the EU that process data of EU citizens as a service to support the effective operation of smart factories.

Furthermore, with personalized manufacturing and product individualization being a very important objective of I4.0, it is obvious that new regulations and directives will be imposed to protect the privacy of individual employee in the near future, making it more complicated in terms of process flow.

\section{Industry 4.0, 5-Layer Architecture}

Here for addressing research problem, work is based on Industry 4.0 concepts for generation and collection of time data from employee daily activities on tasks and other doable activities, mathematical algorithms are used to find further performance parameters and indexes for the decision-making use, for this purpose, we need to get help of IoT, smart gadgets, and a software application to interpret and record data.

\section{5-Layer}

The 5-Layer architecture of CPS system, Industry 4.0 which should be applied to the monitoring and control in project management are elaborated below:

Smart connections: (to acquire accurate and reliable data). Acquisition of accurate and reliable data from devices is the first step in deploying CPS system application for implementation of Industry 4.0. Data acquisition of time utilized by employee in project and tasks is a starting factor or base of success, because without a reliable data no outcomes are possible. Selection of proper device is very important and very critical, such kind of device which can record time of different activities in accordance is very important.

Data to information conversion. Meaningful information must be inferred from the data. Currently, there are several tools and methodologies available for the data to information conversion level. In recent years, extensive focus has been applied to develop these algorithms specifically for prognostics and health management applications. By calculating health value, estimated remaining useful life etc., the second level of CPS architecture brings self-awareness.

Cyber layer. The cyber layer acts as central information and calculations hub in this architecture. Information is being called from the database. Having information received, specific algorithms and analytics have to be used to extract additional information that provides better insight over the status of every individual employee in the project. These algorithms and analytics provide performance indexes of employee, time utilization graphs, and task performance patterns for predictions and decision-making processes. 
The analysis and results produced in this layer will be presented in graphs and performance reports for quick overview and further interpretation by managers, the analysis, reports, and graphs are stored in database for further utilization. When implemented these algorithms in CPS system, system when received information about inputs can calculate all other parameters and measures by itself and present its reports and interpretation. In this way project control will become as simple as possible. Inputs and data are to be evaluated by CPS system with the help of EVM mathematical algorithms, where time allocation array is the input to the CPS system, while CPS calculates and evaluates the forecast and variances of the project with the help of EVA techniques.

Inputs to the CPS system used for the project or task plan are as:

Table 2

Input Parameters for EVA Based CPS

\begin{tabular}{lll}
\hline Input parameters & Values & Units \\
\hline Total scheduled task (Hrs) & 64 & Hours \\
effort per day & 8 & Hours/day \\
Cost per day of employee & $€ 800.00$ & Per day \\
Cost per hour of employee & $€ 100.00$ & Per hour \\
BAC of assignment & $€ 6,400.00$ & Per task \\
Days to deadline & 8 & Days max. \\
Planned completion date & $3 / 10 / 2019$ & \\
\hline
\end{tabular}

Inputs are provided to the CPS system which is equipped with EVA algorithms with the help of python codes results in the provision of Project health parameters such as Cost performance index, Schedule performance index, time performance index, Earned Value, Earned Schedule and Project forecast including Cost Estimate to Complete, Time Estimate to Complete, and TVAC. The algorithms for the EVA analysis in CPS are given below with step by step incremental patterns as per occurrence in the algorithms towards the final outputs.

$$
\begin{gathered}
\text { Planned } \% \text { work }=\left(\frac{\text { scheduled activity for day }}{\text { total scheduled Task }}\right) \\
\text { Budget Work }(B W)=\% P W / \text { day } * \text { Total } P W \\
\text { Actual Work } \%=\left(\frac{\text { actual activity per day }}{\text { total scheduled Task }}\right) \\
\text { Budgeted Cost }=(\% \text { PW } / \text { day } * \text { total Budget }) \\
\text { ACWP }=\left(\frac{\text { Scheduled Activity }}{\text { day }} * \frac{\text { Employeecost }}{h}\right)
\end{gathered}
$$

Earned Value $=($ Tot. Budget Cost $* \% A W)$ 


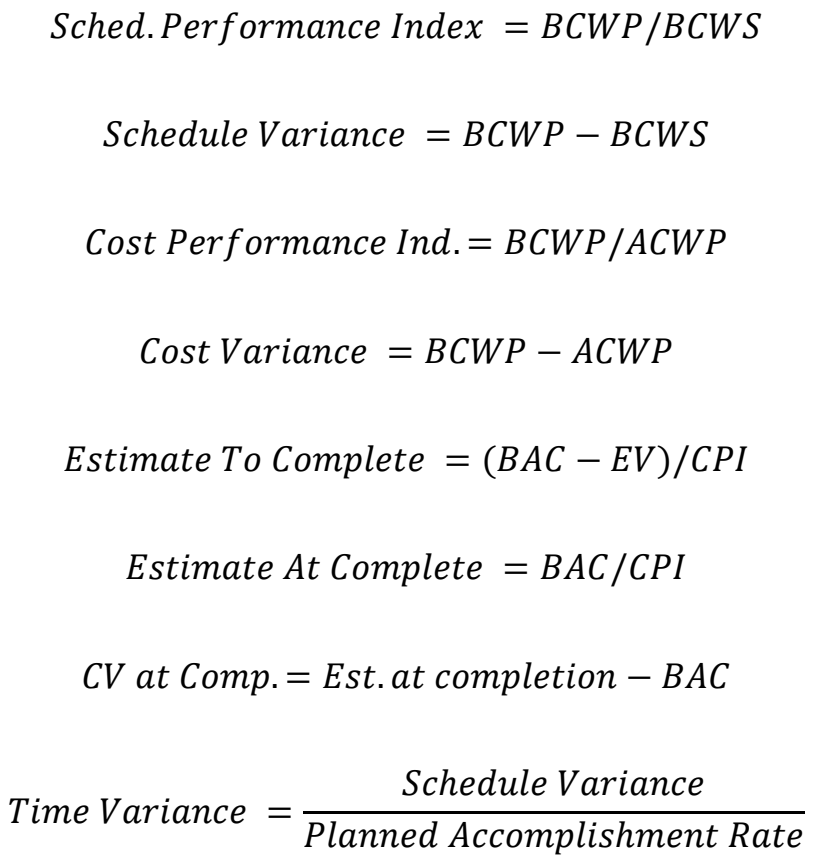

$$
\begin{gathered}
\text { Time Variance } \%=\frac{\text { Time } \text { Varince }}{B W} \\
\text { Earned Schedule }=\frac{\text { Earned Value }}{P A R} \\
\text { Time Performance Index. }=\frac{\text { Earned Schedule }}{B W} \\
\text { Estimated Accomplishment Rate }=E V / B W \\
\text { Time Estimate At Comp. }=B W+\frac{B A C-E V}{E A R} \\
\text { Time Variance At Comp. }=B W-T E A C
\end{gathered}
$$

The final outputs are the EV, CPI, SPI, SV, CV, TEAC, TPI, TVAC and Earned Schedule. Table 2 shows the inputs and the outputs of the system in the same fashion.

The EVA based Python code for the implementation in CPS based on above discussed algorithms as white box twin testing is presented in the separate section below. 
Table 3

CPS System Algorithms for Analysis and Forecasting of a Task or Project

\begin{tabular}{|c|c|c|c|c|c|c|c|c|c|c|c|}
\hline Days & $\begin{array}{l}\text { Schd. } \\
\text { activity/day }\end{array}$ & $\begin{array}{l}\text { Actual } \\
\text { activity/day }\end{array}$ & Unit & $\begin{array}{l}\mathrm{BW} \\
\text { (Hrs) }\end{array}$ & $\begin{array}{l}\mathrm{AW} \\
\text { (Hrs) }\end{array}$ & EV & SPI & CPI & $\begin{array}{l}\mathrm{CV} \text { at } \\
\text { completioneuro }\end{array}$ & TPI & $\begin{array}{l}\text { TVAC } \\
\text { (Hrs) }\end{array}$ \\
\hline 1 & 8 & 4 & hrs & 8 & 4 & $€ 400.00$ & 0.5 & 0.5 & $€ 800.00$ & 0.5 & -8.0 \\
\hline 2 & 8 & 5 & hrs & 16 & 9 & $€ 900.00$ & 0.5625 & 0.5625 & $€ 1,244.44$ & 0.5625 & -12.4 \\
\hline 3 & 8 & 3 & hrs & 24 & 12 & $€ 1,200.00$ & 0.5 & 0.5 & $€ 2,400.00$ & 0.5 & -24.0 \\
\hline 4 & 8 & 4 & hrs & 32 & 16 & $€ 1,600.00$ & 0.5 & 0.5 & $€ 3,200.00$ & 0.5 & -32.0 \\
\hline 5 & 8 & 5 & hrs & 40 & 21 & $€ 2,100.00$ & 0.525 & 0.525 & $€ 3,619.05$ & 0.525 & -36.2 \\
\hline 6 & 8 & 7 & hrs & 48 & 28 & $€ 2,800.00$ & 0.5833333 & 0.5833 & $€ 3,428.57$ & 0.583333333 & -34.3 \\
\hline 7 & 8 & 6 & hrs & 56 & 34 & $€ 3,400.00$ & 0.6071429 & 0.6071 & $€ 3,623.53$ & 0.607142857 & -36.2 \\
\hline \multirow[t]{2}{*}{8} & 8 & 3 & hrs & 64 & 37 & $€ 3,700.00$ & 0.578125 & 0.5781 & $€ 4,670.27$ & 0.578125 & -46.7 \\
\hline & 64 & 37 & & & & & & 0.5445 & & & \\
\hline
\end{tabular}

Cognition layer. Implementing CPS upon this level generates a thorough knowledge of the monitored tasks. Proper presentation of the acquired knowledge to expert users supports the correct decision to be taken. Since comparative information as well as individual tasks status is available, decision on priority of tasks to optimize the controlling activities can be made. For this level, proper info-graphics are necessary to completely transfer acquired knowledge to the users.

In this level, performance of a single task can be compared with the planned schedules of that task. On the other hand, these analytics may be used to measure and predict the future performance of the employee.

Here project manager or decision maker can access the performance indexes, reports and graphical representation of productivity of employee generated by cyber layer to understand the patterns and effectiveness of employee, in this way managers are able to make correct decisions.

Configuration layer. The configuration level is the feedback from cyber space to physical space and acts as supervisory control to make tasks satisfactory. This stage acts as supervisory control to apply the corrective and preventive decisions, which have been made in cognition level, to the monitored tasks.

The decisions made in the cognition layer by managers on the basis of reports and graphs available from the database, are controlled in this layer.

Based on performance analyzed by system, manager can act accordingly to control the project.

\section{Business Process Modeling and Notation (BPMN) or Business Process Orchestration}

Business Process Orchestration can simply be defined as the coordination of events and activities in a process at technical levels, to help achieve objectives laid down by the business. The automation of workload can be taken to the next level with business orchestration. This is the process of coordinating and bringing together a wide range of automated tasks into larger, perfectly optimized series of the workflow. Simply put, cloud orchestration makes sure that all automated segments of your business work in harmony. It is useful in improved user experience, agility, minimized cost of operations, transparency and cooperation. Business Process Model (BPM) describes the behavioral aspects of a system on formal or early design levels.

BPMN is graphical representation for the purpose to summarize business processes in the form of model and notations, so that process can be understood easily. BPMN is standard for process models in business modeling dedicated for graphical notations to elaborate business process diagram (BPD) to support business process management for the business and technical users. 


\begin{tabular}{|c|c|c|}
\hline TASK NAME & NOTATION & DESCRIPTION \\
\hline Service Task & Service & $\begin{array}{l}\text { Task which uses other services for } \\
\text { the completion of task }\end{array}$ \\
\hline Send Task & Send & $\begin{array}{l}\text { Task which for completion send a } \\
\text { message to acquire necessary } \\
\text { information to a different lane or pool, } \\
\text { and when information is received task } \\
\text { is performed. }\end{array}$ \\
\hline Receive Task & $\square$ Receive & $\begin{array}{l}\text { Which has to be started when a } \\
\text { certain kind of a message is arrived to } \\
\text { it. }\end{array}$ \\
\hline User Task & 3 & $\begin{array}{l}\text { Task that has to be performed by } \\
\text { human, by means of software } \\
\text { application of other tools. }\end{array}$ \\
\hline Manual Task & Manual & $\begin{array}{l}\text { Tasks which are performed without } \\
\text { the help of business process } \\
\text { execution engines or other types of } \\
\text { application }\end{array}$ \\
\hline Business Rule Task & $\begin{array}{c}\text { Business } \\
\text { Rule }\end{array}$ & $\begin{array}{l}\text { Task give input to a business rule } \\
\text { engine and in response the output is } \\
\text { given by BRE }\end{array}$ \\
\hline Script Task & Script & $\begin{array}{l}\text { Task is performed by business } \\
\text { process engine, task defines a script } \\
\text { as evidence from task name that the } \\
\text { engine can read }\end{array}$ \\
\hline
\end{tabular}

Figure 1. BPMN legends and descriptions.

For the process elaborated for employee management and to analyze their performance, BPMN standards are used to specify the model of process with essential notations:

\section{Process Modeling (Process Working Model)}

When I4.0 based CPS system is implemented to project monitoring and control upon employee time monitoring, the system model as elaborated below with the help of Business Process Modeling and Notations techniques and rules, the process is elaborated below:

When in an employee loop, an employee starts to work on an assigned task, he can access his time monitoring device, where he can select a working task which is assigned to him, as soon as task is selected the process proceeds towards the validation of that specific task, for this validation an information request is sent to the system monitoring loop in system group, where task status monitoring system generates an activity of task validation and confirms the status of task, if task selected was an old task, means employee was already working on that task before logging off, task status will generate task of continue countdown, which means task validation in employee loop will generate check-in and start countdown. On the other hand if the task is new means employee logoff was another task, then a parallel fork will be generated, it will send information to task validation for starting new task countdown and check in and employee time tracking loop is terminated on end task. On the other hand task monitoring system will logout old task and save time information to Time Allocation Array Database.

Now Interpreter loop, which consists of CPS system, receives Time Allocation Array information from the database and starts data analysis based on pre designed mathematical algorithms for analysis, when analysis is done, reports and graphs are generated, which are sent to database for further analysis by manager. 


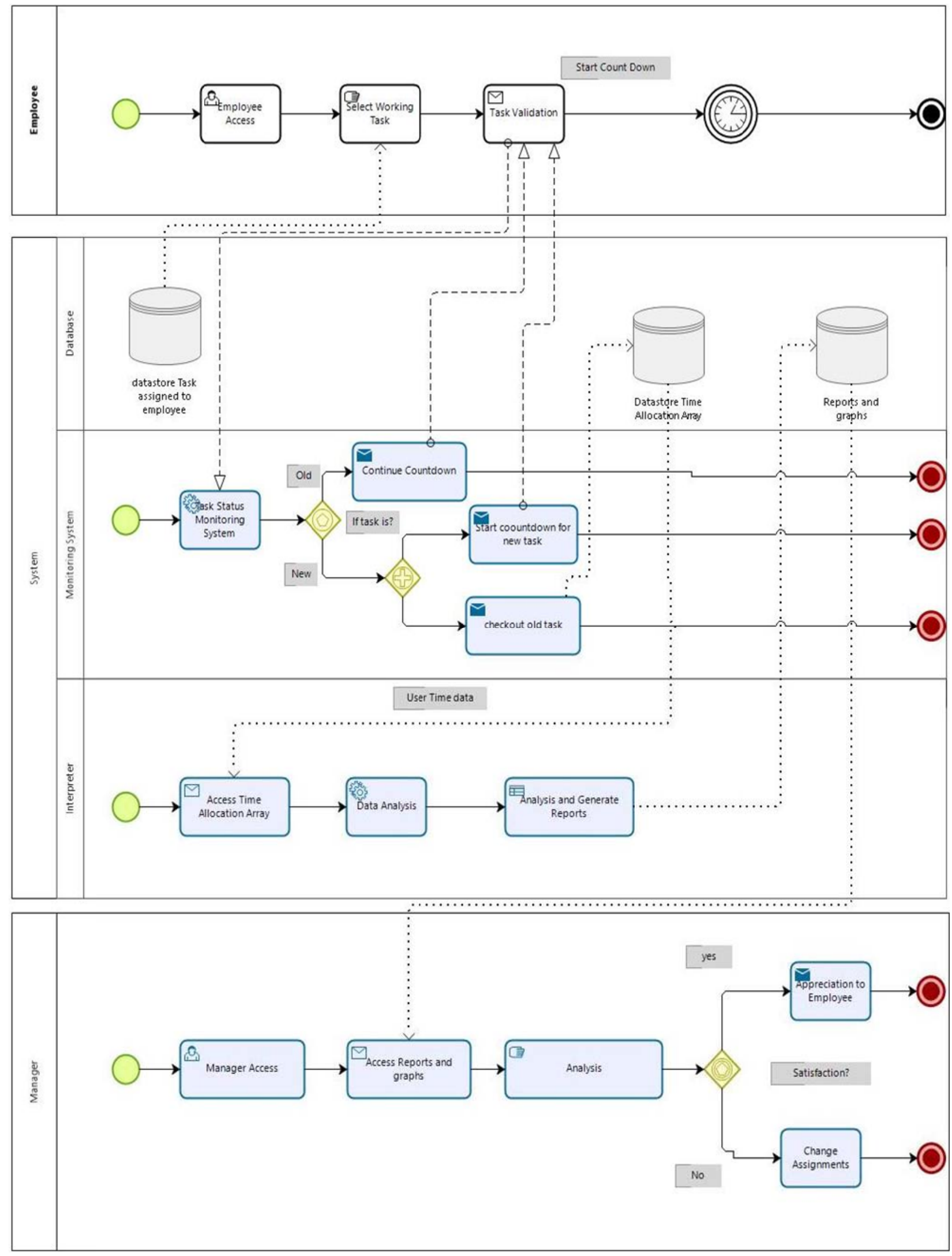

Figure 2. BPMN (Business Process Model for CPS system). 
Manager loop starts when manager access generated reports and graphs for review and decide if employee performance is satisfactory, an appreciation is sent to employee, or performance is not satisfactory then employee is subject to relocation to another task.

There are several tasks and their specific notations which are used in this model, according to BPMN standards. Given below are details of tasks.

\section{Python Code for EVA Based CPS System-White Box Testing}

(Python Coding for Mathematical Algorithms, CPS system)

from datetime import date

import matplotlib.pyplot as plt

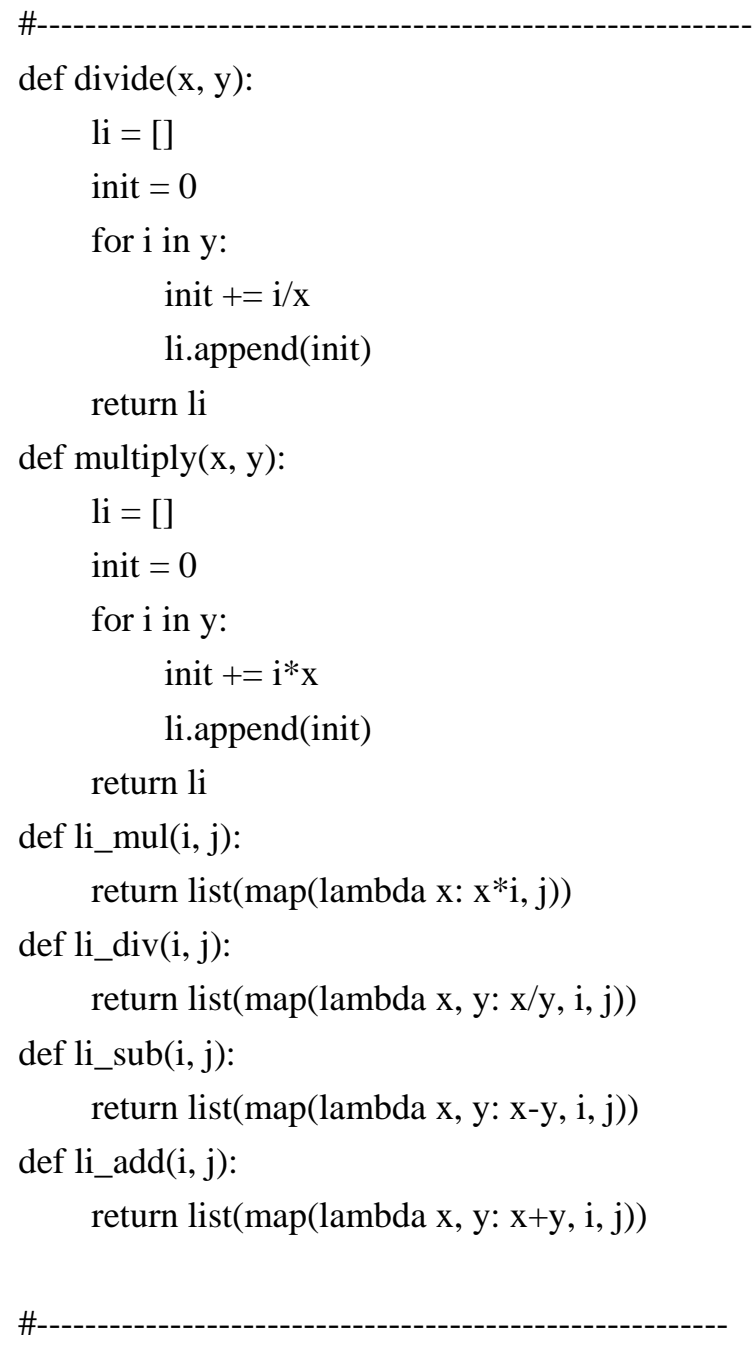

taskDuration $=8$

\# Duration of Task to be completed in days

dateOfAllocation $=$ date $(2019,9,24)$

totalScheduleTask $=64$ 
\#in hours

effortPerDay $=$ int(totalScheduleTask/taskDuration)

employeeCostDay $=800$

\#rough estimate

employeeCostHour $=$ employeeCostDay/effortPerDay

\#based on 8 hours normal office working hour

bac $=$ employeeCostHour*effortPerDay*taskDuration

\#per task

days $=$ list(range $(1$, taskDuration+1) $)$

scheduledActivity = [effortPerDay for i in range(taskDuration)]

\#schedule time array for task completion

actualActivity $=[4,5,3,4,5,7,6,3]$

\#in hours, actual workout on task by employee, time tracking devices can be used to monitor for activity of task, as well as some autonomous devices which can also be used which tracks activity and record it in the system automatically with the help of coding and synchronization

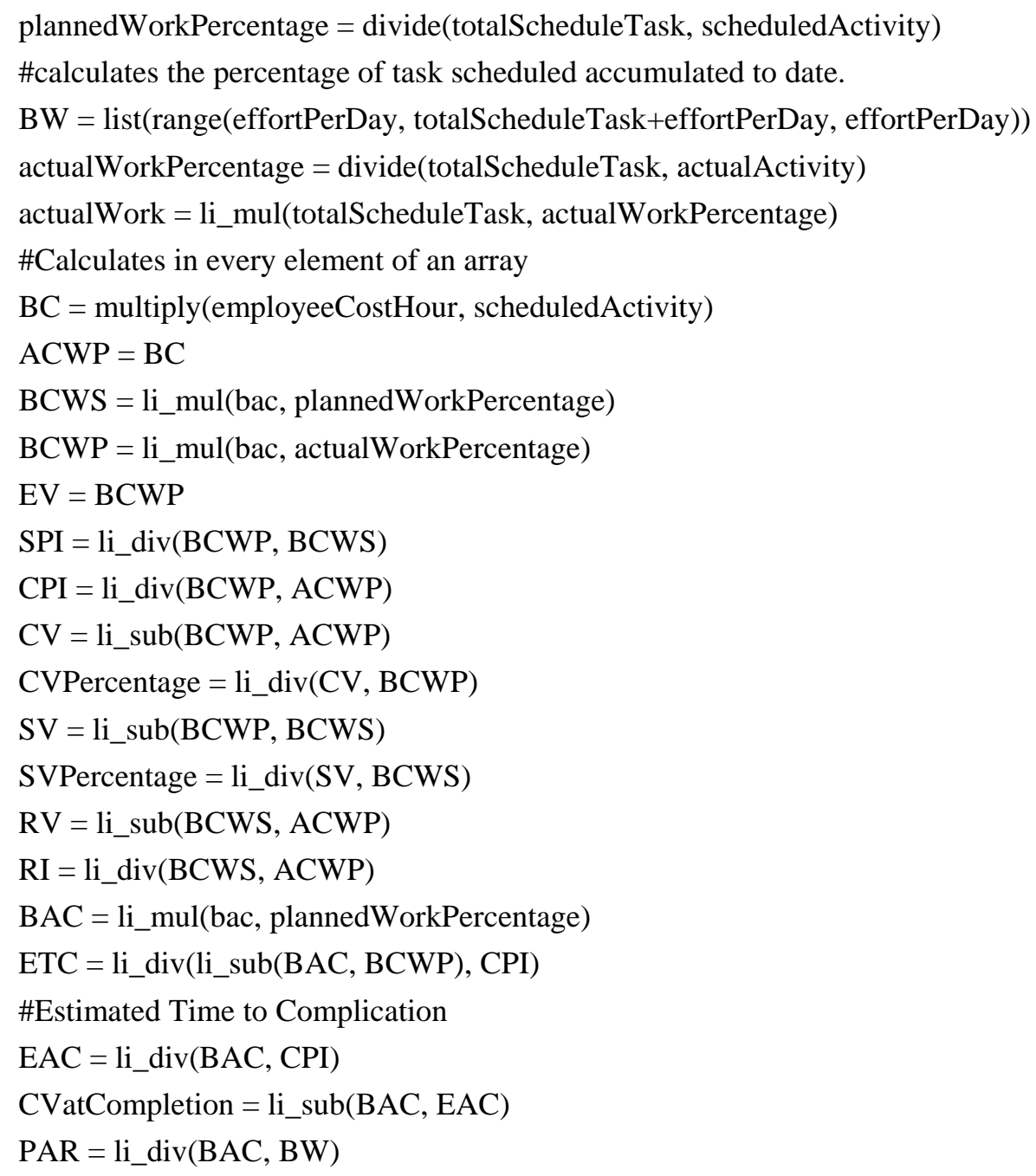


$\mathrm{TV}=$ li_div(SV, PAR)

TVPercentage $=$ li_div $(T V, B W)$

ES = li_div(EV, PAR)

TPI $=$ li_div(ES, BW)

EAR = li_div(BCWP, BW)

TEAC $=$ li_add(BW, li_div(li_sub(BAC, BCWP), EAR))

TVAC $=$ li_sub $($ TEAC, BW)

\#Employee performance individually based on accumulated and each day activity, irrespective of the project performance

\# Overall Evaluation

evaluation $=6$

employeePerformance $=\operatorname{sum}($ actualActivity[0:evaluation])/sum(scheduledActivity[0:evaluation])

statusOfTask $=$ ('Task is Behind Schedule' if (employeePerformance $<1)$ else 'Task is On Time' if (employeePerformance ==1) else 'Task is Ahead of Schedule')

delta $=$ abs(employeePerformance- 1 )

estimatedTimeOfCompletion $=$ taskDuration* $(1+$ delta $)$

statusAccordingToDeadline $=$ taskDuration-estimatedTimeOfCompletion

\#Performance Evaluation of Single Day

daysOfEvaluation $=5$

effortOnTheDay = actualActivity[daysOfEvaluation-1]

effortPerDayRequired = effortPerDay

performanceEvaluation = effortOnTheDay/effortPerDayRequired

statusOfCurrentDay $=$ statusOfTask $=$ ('Behind Schedule' if $($ performanceEvaluation $<1)$ else 'On Schedule' if (employeePerformance == 1) else 'Ahead of Schedule')

\# Plots

plt.figure()

plt.subplot(211)

plt.plot(days, ACWP, days, BCWP, days, BCWS)

plt.title('Earned Value Graph')

plt.xlabel('Duration')

plt.ylabel('Cost')

plt.subplot(212)

plt.plot(days, SPI)

plt.axis([0, taskDuration $+1,0,0.7])$

plt.title('Performance Chart')

plt.xlabel('Duration') 


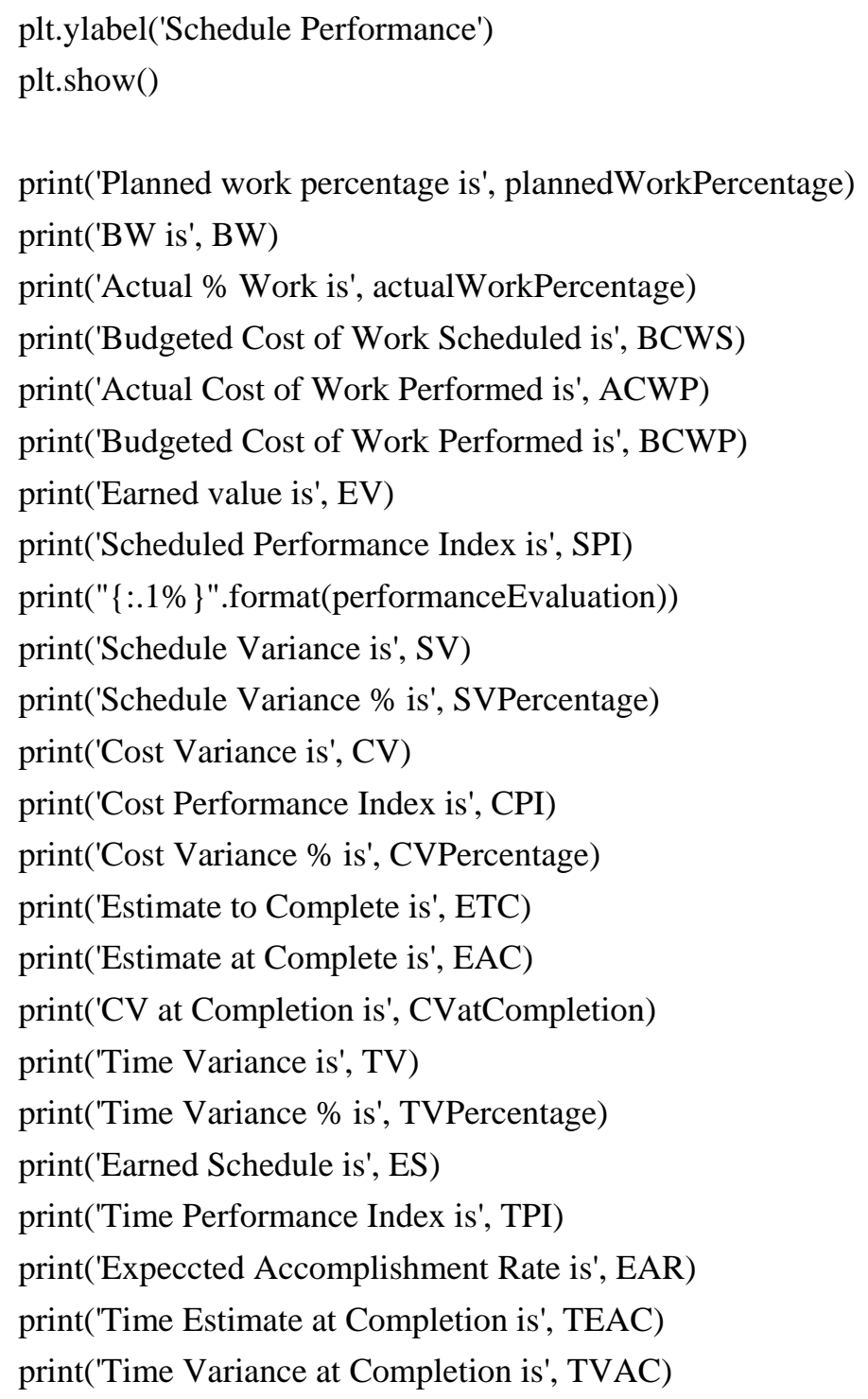

print('for task duration', taskDuration, 'allocated on', dateOfAllocation, 'total schedule of activity (hours)', totalScheduleTask, 'and effort per day as respected to total schedule is', effortPerDay, 'per day cost of nominated employee is', employeeCostDay, 'so per hour cost of working on this task is', employeeCostHour, 'in this response total schduled budget of task is', bac)

print('for schedules per day as', scheduledActivity, 'employee workout on activity as', atualActivity, 'so according to analysis, Eatimate to complete of the project will be', ETC, 'while estimate at complete will be', EAC, 'while time estimate at compeion will be', TEAC)

\section{Conclusions}

This study arrives at the conclusion that if proper devices for the acquisition of correct project data are developed and the earned value analysis algorithms are utilized in the cyber physical system for the analysis of data, we clam that Industry 4.0 paradigms are feasible to be implemented in project management. And that this 
implementation will come with a certain level of automation in decision making and accuracy in the project forecasts.

According to the literature review these concepts were never discussed in these details, this research study elaborated every step in detail for the implementation of automation trend in project management for the advancement of the field.

Further research in this domain can also be conducted for the optimization of the solution and implementation to the other areas of project management.

\section{Limitations and Implications}

There are some limitations to this research. Work elaborated in this research work has not been tested, in order to get real results. For the acquisition of data no physical sensors or devices have been used in order to get real data, instead considered data values have been utilized to check the feasibility and meaningfulness of the concepts for the field. Data have been considered randomly keeping in mind the actual average behavior of employee working in enterprises on fixed unit income allocation basis.

Keeping in consideration the rules and regulations imposed by European General Data Protection Regulations, during the study of this research employee we had resources assessing personal data of employee or employee time allocation on tasks during projects with the help of data acquisition devices. The GDPR significantly affects businesses in all industry sectors, including those outside the EU that process data of EU citizens as a service to support the effective operation of smart factories.

Furthermore, as of limitations of the Project management with I4.0 it is expected that General Data Protection Regulations (GDPR) may put some more regulatory borders on accessing some employee data from devices during project activities ensuring the privacy of workers because of personalized manufacturing and product individualization being a very important objective of I4.0, it is obvious that new regulations and directives will be imposed to protect the privacy of individual employee in the near future, making it more complicated in terms of process flow.

Implications of the approach are the architecture of ICT infrastructure for the implementation of Industry 4.0 to project management for monitoring and control.

\section{Reference}

Anbari, F. (2003). Earned value project management method and extensions. Project Management Journal, 34, 12-23.

Danielle, R. (2016). 21 Shocking project management statistics that cost business owners millions each year. Mavenlink Blog, 2017. Available at https://blog.mavenlink.com/21-shocking-project-management-statistics-that-explain-why-projects-continu e-to-fail

Hunter, H., Fitzgerald, R., \& Barlow, D. (2014). Improved cost monitoring and control through the Earned Value Management System. Acta Astronautica, 93, 497-500.

Kolberg, D., \& Zuhlke, D. (2015). Lean automation enabled by industry 4.0 technologies. IFAC-PapersOnLine, 48(3), 1870-1875.

Lee, J., Bagheri, B., \& Hung-An, K. (2014). A cyber-physical systems architecture for industry 4.0-based manufacturing systems. Manufacturing Letters, 3,18-23.

Li, Z., Wang, Y., \& Wang, K. S. (2017). Intelligent predictive maintenance for fault diagnosis and prognosis in machine centers: Industry 4.0 scenario. Advances in Manufacturing, 5(4), 377-387.

Mahadik, S. G., \& Bhangale, P. P. (2013). Study \& analysis of construction project management with earn value management system. International Journal of Innovative Technology and Exploring Engineering, 3.

Müller, J. M., Kiel, D., \& Voigt, K. I. (2018). What drives the implementation of industry 4.0? The role of opportunities and challenges in the context of sustainability. Sustainability, 10(1), 247. 
Narbaev, T., \& De Marco, A. (2013). Combination of growth model and earned schedule to forecast project cost at completion. Journal of Construction Engineering and Management, 140(1).

Oesterreich, T. D., \& Teuteberg, F. (2016). Understanding the implications of digitization and automation in the context of Industry 4.0: A triangulation approach and elements of a research agenda for the construction industry. Computers in Industry, 83, 121-139. https://doi.org/10.1016/j.compind.2016.09.006

Petrasch, R., \& Hentschke, R. (2016). Process modeling for industry 4.0 applications: Towards an industry 4.0 process modeling language and method. 13th International Joint Conference on Computer Science and Software Engineering (JCSSE).

Preuveneers, D., \& Ilie-Zudor, E. (2017). The intelligent industry of the future: A survey on emerging trends, research challenges and opportunities in Industry 4.0. Journal of Ambient Intelligence and Smart Environments, 9(3), 287-298. DOI 10.3233/AIS-170432

Strange, R., \& Zucchella, A. (2017). Industry 4.0, global value chains and international business. Multinational Business Review, 25(3), 174-184. ISSN 1525-383X. This version is available from Sussex Research Online: http://sro.sussex.ac.uk/id/eprint/68111/

Theorin, A., Bengtsson, K., Provost, J., Lieder, M., Johnsson, C., Lundholm, T., \& Lennartson, B. (2017). An event-driven manufacturing information system architecture for Industry 4.0. International Journal of Production Research, 55(5), 1297-1311. DOI: 10.1080/00207543.2016.1201604

Win, T. Z., \& Kham, N. S. M. (2018). Transformation of project management in industry 4.0. 